Presented at ICCV’99, Kerkyra, 1999.

\title{
Bayesian Fusion of Color and Texture Segmentations
}

\author{
Roberto Manduchi \\ Jet Propulsion Laboratory \\ California Institute of Technology \\ Pasadena, CA 91109 \\ manduchi@jpl.nasa.gov
}

\begin{abstract}
In many applications one would like to use information from both color and texture features in order to segment an image. We propose a novel technique to combine "soft" segmentations computed for two or more features independently. Our algorithm merges models according to a maximum descriptiveness criterion, and allows to choose any number of classes for the final grouping. This technique also allows to improve the quality of supervised classification based on one feature (e.g. color) by merging information from unsupervised segmentation based on another feature (e.g., texture.)
\end{abstract}

\section{Introduction}

Image segmentation is a fundamental task in Computer Vision. Color and texture provide powerful cues for segmenting a still image, and much work has been devoted to developing grouping algorithms based on these two features [1],[3],[5]. In fact, most of the literature deals with segmentation based on either color or texture; this work was originated by the intuition that using information provided by both features, one should be able to obtain more robust and meaningful results.

Underlying our approach is the hypothesis that in typical images color and texture features are not statistically independent. Perhaps the simplest way to exploit this dependency is to concatenate the color and texture feature vectors together, and then run the grouping algorithm of choice on such super-vectors. This approach, however, may give the feeling of "comparing apples with oranges". Indeed, color and texture features often have very different statistical behaviors; one may prefer to use the most suitable grouping algorithm for each feature separately, and then somehow combine the results of the two segmentations together.

This work introduces a strategy to merge together in a Bayesian framework segmentations computed on color and texture features independently. The only requirement is that the segmentations are expressed in terms of posterior probabilities [2]. Note that most clustering algorithms explicitly compute estimates of the posterior distributions, and do the final assignment by Bayesian classification (i.e., they assign a feature to the class that most likely generated it.)

For example, in Figure 2 (b) and (c) we show instances of color and texture segmentation of the image in Figure 2 (a). The texture features are vectors formed by the absolute values of the outputs of a bank of Gabor filters, after smoothing by a gaussian filter [3]. The posterior distributions in both cases have been estimated by Expectation Maximization [2]; the "hard" segmentation shown in the figures is the result of Bayesian classification based on such distributions. Both models have four classes, although our algorithm can accept any combination of classes. The scene in figure 2(a) is composed by a small number of homogeneous parts: two bushes, a paved road on the right, dirt soil on the left, a shadow area near a bush and piece of dark background. The color segmenter (figure 2(b)) successfully separates the "bush", the "background" and the "road" areas, but is unable to discriminate the "road" from "soil" parts, which have very similar color. The texture segmenter does separate the "road" and "soil" areas, but cannot discriminate the "road" from the "background" parts; in addition, it assigns the "soil" area to two distinct classes.

Our technique for model fusion involves two steps. First, the two models are merged by a "Cartesian product" operator, discussed in section 2. This operation preserves all the information about the models, but has the disadvantage of creating a large number of classes, equal to the product of the number of classes of the two original models. Then, the number of classes of the combined model is reduced by a technique, pre- 
sented in section 3, that "clips together" sets of classes based on a maximum descriptiveness criterion. This procedure may be extended straightforwardly to any number $K$ of segmentations. An intriguing application of our algorithm is discussed in section 4, and involves information fusion from supervised classification (e.g., based on color) and unsupervised segmentation (e.g., based on texture.) The unsupervised segmentation is used to leverage the estimates provided by the trained model, resulting in a more accurate classification.

\section{Cartesian product of mixture models}

Our merging technique starts from $K$ given mixture models [2] (called "models" in the following.) The $i$-th model, $\mathcal{M}_{i}$, is composed by $N_{i}$ classes, and defines a probability density function $p_{i}\left(z_{i}\right)$ :

$$
p_{i}\left(z_{i}\right)=\sum_{j_{i}=1}^{N_{i}} p_{i}\left(z_{i} \mid j_{i}\right) P_{i}\left(j_{i}\right)
$$

where $z_{i}$, the observed feature, lives in a space $Z_{i}$. For example, $z_{i}$ may be a color vector, or a texture feature in a multiscale/multiorientation space. The conditional likelihood functions $p_{i}\left(z_{i} \mid j_{i}\right)$ and the priors $P_{i}\left(j_{i}\right)$ specify the model completely. The posterior distributions are given by Bayes' rule:

$$
P_{i}\left(j_{i} \mid z_{i}\right)=\frac{p_{i}\left(z_{i} \mid j_{i}\right) P_{i}\left(j_{i}\right)}{p_{i}\left(z_{i}\right)}
$$

$P_{i}\left(j_{i} \mid z_{i}\right)$ is the probability that the observed feature $z_{i}$ was generated by the class of index $j_{i}$. The Bayesian classifier for $\mathcal{M}_{i}$ assigns a feature $z_{i}$ to the class indexed by the location of the maximum of $P_{i}\left(j_{i} \mid z_{i}\right)$. To simplify our presentation, we will assume in the following that all priors are strictly positive: if a prior $P_{i}\left(j_{i}\right)$ is null, we can safely remove the class with index $j_{i}$ from the model. Note that

$$
P_{i}\left(j_{i}\right)=\int P_{i}\left(j_{i} \mid z_{i}\right) p_{i}\left(z_{i}\right) d z_{i}=E\left[P_{i}\left(j_{i} \mid z_{i}\right)\right]
$$

where the expectation is computed with respect to the density $p_{i}\left(z_{i}\right)$.

The Cartesian product $\mathcal{M}$ of the models $\mathcal{M}_{i}$ is a new model with probability distribution $Z_{1} \times \ldots \times Z_{N}$. $\mathcal{M}$ is completely specified by the following axioms:

1. $\mathcal{M}$ has $N=\prod_{i=1}^{K} N_{i}$ classes, corresponding to the Cartesian product of the classes of the models $\mathcal{M}_{i}: j \leftrightarrow\left(j_{1}, \ldots, j_{K}\right)$.

2. The conditional likelihood of the feature $z=$ $\left(z_{1}, \ldots, z_{K}\right)$ given the class of index $j$ is equal to $p(z \mid j)=\prod_{i=1}^{K} p_{i}\left(z_{i} \mid j_{i}\right)$.
3. The priors factorize as $P(j)=\prod_{i=1}^{K} P_{i}\left(j_{i}\right)$.

It follows straightforwardly that the likelihood and the posteriors of the Cartesian product of models factorize as well:

$$
p(z)=\prod_{i=1}^{K} p_{i}\left(z_{i}\right), P(j \mid z)=\prod_{i=1}^{K} P_{i}\left(j_{i} \mid z_{i}\right)
$$

Note that all the information about the $K$ original models is preserved in their Cartesian product $\mathcal{M}$. The Bayesian classifier for $\mathcal{M}$ assigns a feature $z$ to the model $j \leftrightarrow\left(j_{1}, \ldots, j_{N}\right)$ such that $j_{i}$ is the class assigned to $z_{i}$ by the Bayesian classifier for $\mathcal{M}_{i}$. Figure 2 (d) shows the Bayesian segmentation relative to the Cartesian product of the color and texture models of figure 2 (b) and (c). The new model has 16 classes. In the next section we describe a procedure to reduce the dimensionality (i.e., the number of classes) of a model, in such a way that the loss of "descriptiveness" of the model is minimized.

\section{Dimensionality reduction}

Assume we are given a model $\mathcal{M}$ with $N$ classes. We introduce here a technique to build a new model that has fewer classes than $\mathcal{M}$ but explains the data exactly as $\mathcal{M}$, i.e., it defines the same likelihood $p(z)$ as $\mathcal{M}$. Suppose for example that we want to reduce the dimensionality of the model to $N-M$. Our strategy is very simple: we just "clip together" $M+1$ classes of $\mathcal{M}$ into a new super-class, leaving the other classes untouched. We may decide, for instance, to clip together the classes of index $N-M, \ldots, N$ into a new class of index $N-M$. The probability that a feature $z$ was generated by the union of such classes according to $\mathcal{M}$ is equal to the sum of the corresponding posteriors. This is the value that we assign to the posterior $P^{n e w}(N-M \mid z)$ for the new model; the posteriors for the other classes are the same as in $\mathcal{M}$ :

$$
\begin{aligned}
& P^{n e w}(j \mid z)=P(j \mid z), 1 \leq j<N-M \\
& P^{n e w}(N-M \mid z)=\sum_{j=N-M}^{N} P(j \mid z)
\end{aligned}
$$

If in addition we impose that the likelihood function $p(z)$ is the same in both models, the new model is completely specified.

In general, to reduce the model dimension from $N$ to $N-M$, we may choose any $L \leq M$ disjoint groups of classes with $L_{l}$ components each, such that $\sum_{l=1}^{L} L_{l}=$ $L+M$, and clip together the classes in each group. A criterion for the selection of the most appropriate clipping scheme is presented in the next section. 


\subsection{Maximum descriptiveness criterion}

Dimensionality reduction via class-clipping involves some loss of descriptiveness of the model, where by "descriptiveness" we mean the information that the model provides about the image. If for example two classes that "explain" well two different portions of the image are clipped together, the new, less informative model will probably assign both portions of the image to the same class. In this section we give a formal definition of descriptiveness, and present an algorithm for selecting a class-clipping scheme that minimizes the loss of descriptiveness for a given model.

Loosely speaking, we will say that a model is highly descriptive if its classes "explain well" the features that are assigned to them. More precisely, we define the descriptiveness $D(j)$ of class $j$ as follows:

$$
D(j)=\int P(j \mid z) p(z \mid j) d z
$$

while the descriptiveness of the model, $D$, is the sum of the class descriptivenesses:

$$
D=\sum_{j=1}^{N} D(j)
$$

Thus, the class descriptiveness $D(j)$ is the integral of the conditional likelihood weighted by the posterior distribution. It is clear from $(5)$ that $0<D(j) \leq 1$ (since $\int p(z \mid j) d z=1, p(z \mid j) \geq 0$ and $0 \leq P(j \mid z) \leq 1$ ) and therefore $D \leq N$. A single-class model has $D=1$, which is the smallest value of descriptiveness attainable by a model (this property derives straightforwardly from Fact 1, presented later in this section.)

To justify our choice for the descriptiveness, let us consider two diametrically different examples of models with two classes. In the first model, the two posterior probabilities have disjoint supports in feature space. Each class thus completely describes (by means of the corresponding conditional likelihood) the set of features that are assigned to it. It is easily seen that $D(j)=1$ for both classes, and therefore the model descriptiveness $D$ is equal to 2 , the highest attainable value for a two-class model. It is intuitive that clipping together these two classes would result in a major loss of information (descriptiveness) of the model. The corresponding variation of descriptiveness is actually $\Delta D=-1$. In the second model, the two classes have exactly the same conditional likelihood and priors (and therefore the same posteriors $P(j \mid z)=0.5$.) This model is "redundant": there is really no need to use two classes to describe the data! No information is lost if such two classes are clipped together. This notion is captured by our definition of descriptiveness, that assigns $D=1$ to the model. Class-clipping thus gives $\Delta D=0$ in this case.

In both previous examples the model descriptiveness did not increase as a consequence of class clipping. This is actually a general property of descriptiveness, as stated by the following result (whose proof can be found in the Appendix):

Fact 1 Class-clipping never increases the descriptiveness of a model.

We thus propose the following criterion for dimensionality reduction: choose the clipping scheme that minimizes the decrement of the model descriptiveness.

Unfortunately, the number of possible clipping schemes may be very high even for small model dimensions. For example, in order to reduce the number of classes from 16 to 13 we may choose among 165,620 different combinations of class clipping. Measuring the decrement of model descriptiveness for each one of those schemes may require a prohibitive computational cost. A suboptimal solution can be found using a fast greedy algorithm that builds a sequence of clippings involving only two classes at a time. At each step, the two classes that minimize the decrement of model descriptiveness are selected. To compute the model descriptiveness, we make use of the following identity (from (5) and using Bayes' rule):

$$
\begin{aligned}
D(j) & =\int P(j \mid z) \frac{P(j \mid z) p(z)}{P(j)} d z \\
& =\frac{E\left[P(j \mid z)^{2}\right]}{P(j)}
\end{aligned}
$$

where the expectation is computed with respect to $p(z)$. In practice, the expectations in $(7)$ are estimated by averaging $P(j \mid z)^{2}$ over the image. Our greedy algorithm for class-clipping is described in detail in figure 1.

Figure 2 (e)-(i) shows the results of Bayesian segmentation after dimensionality reduction from 16 to $7,6,5,4$ and 3 classes respectively, based on our maximum descriptiveness criterion. Each class of the reduced dimension models now correctly represents a characteristic area of the image (compare for example figure 2 (h) with (b) and (c) for the 4-class model). The computation of the optimal clipping scheme for reducing the model dimension from 16 to 4, using a Matlab implementation of our greedy algorithm, requires about 15 seconds of execution time on a Power Mac G3 $266 \mathrm{Mhz}$ (the image size is $256 \times 380$ pixels.)

In figure $2(\mathrm{k})$ we plotted the variation of model descriptiveness during the class-clipping process for 


\section{Greedy algorithm for dimensionality reduction: $N \rightarrow N-M$}

Given the set of posteriors $P(j \mid z)$ and of priors $P(j), 1 \leq j \leq N$ :

Build auxiliary vector $\mathbf{D}$ and matrix $\boldsymbol{\Delta}$ :

$\mathrm{D}[j]=\frac{E\left[P(j \mid x)^{2}\right]}{P(j)}, 1 \leq j \leq N$ :

$\Delta[j, k]=\left\{\begin{array}{cl}\mathrm{D}[j]+\mathrm{D}[k]- & \\ -\frac{E\left[(P(j \mid x)+P(k \mid x))^{2}\right]}{P(j)+P(k)} & , 1 \leq k<j \leq N \\ \infty & , \text { otherwise }\end{array}\right.$

Initialize an empty list $L$;

Repeat $M$ times:

$(\bar{j}, \bar{k})=\arg \min \boldsymbol{\Delta}[j, k]$;

Add $\bar{k}$ to the list $L$;

Update $P(\bar{j} \mid z) \leftarrow P(\bar{j} \mid z)+P(\bar{k} \mid z), P(\bar{k} \mid z) \leftarrow 0$;

Update $P(\bar{j}) \leftarrow P(\bar{j})+P(\bar{k})$;

Update $\mathbf{D}[\bar{j}]$;

Update $\boldsymbol{\Delta}[\bar{j}, k]$ for $k<\bar{j}, k \notin L$;

Update $\boldsymbol{\Delta}[k, \bar{j}]$ for $k>\bar{j}, k \notin L$;

Set $\Delta[j, \bar{k}]=\infty$ for $j>\bar{k}$;

Set $\Delta[\bar{k}, j]=\infty$ for $j<\bar{k}$;

Remove the classes indexed by the elements of $L$.

Figure 1: The greedy algorithm to select a classclipping scheme that minimizes the decrement of model descriptiveness (see section 3.1.)

our example (for each model dimension we plotted the (negative) increment $\Delta D$ consequent to the 2-class clipping that generated that model.) Note that the algorithm for the greedy selection of classes, which reduces the dimension by one at a time, allows us to easily compute these values as a by-product. From figure $2(\mathrm{k})$ we notice that the decrement of descriptiveness $(-\Delta D)$ usually increases as the dimension of the model decreases (remember that our algorithm chooses for each dimension the class-clipping that gives the smallest value of $-\Delta D$.) Future work will be devoted to studying the possibility of selecting the "most appropriate" number of classes for the final segmentation based on the analysis of the model descriptiveness behavior.

\subsection{Equalization}

In the previous sections we have described a strategy for model fusion that first builds the Cartesian product of two models, and then performs dimensionality reduction via class-clipping. An implicit assumptions was that the two original models should give the

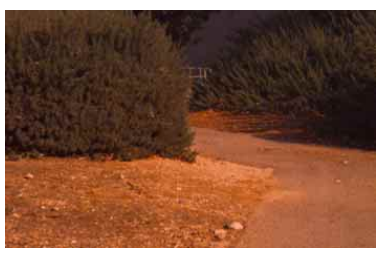

(a)

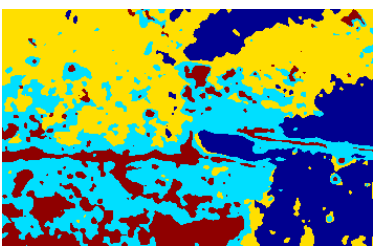

(c)

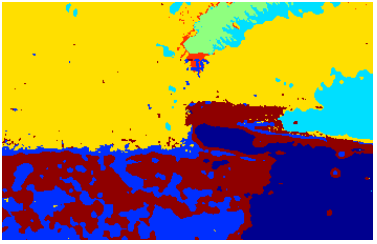

(e)

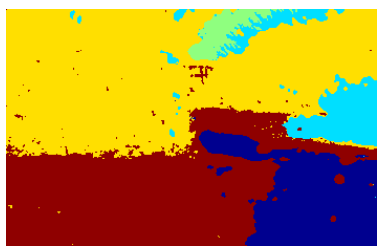

(g)

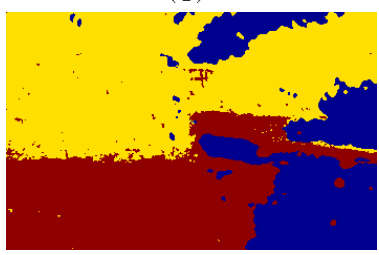

(i)

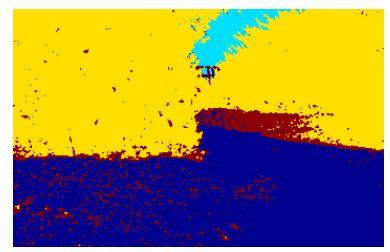

(b)

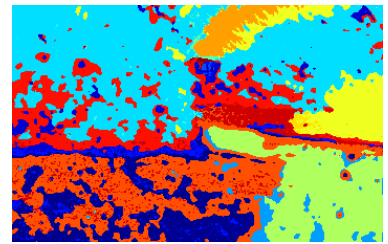

(d)

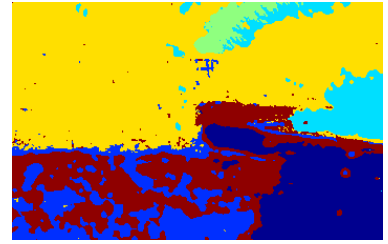

(f)

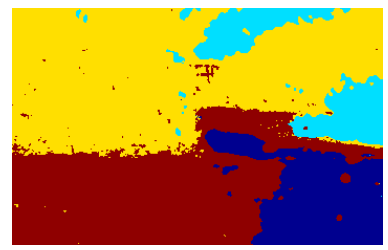

(h)

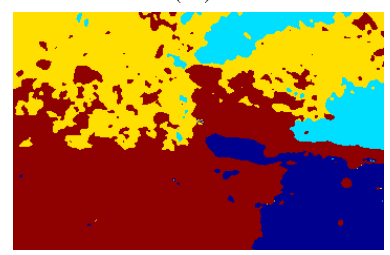

(j)

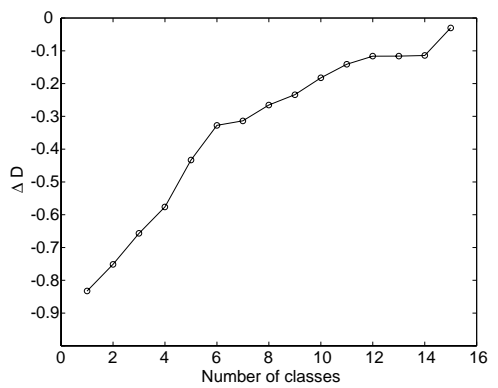

(k)

Figure 2: (a): Test image. (b) Color-based segmentation (4 classes.) (c) Texture-based segmentation (4 classes.) (d) Segmentation after Cartesian product (16 classes.) (e)-(i): Segmentation after model merging ((e): 7 classes, (f): 6 classes, (g): 5 classes, (h): 4 classes, (i): 3 classes.) (j): Segmentation after model merging ( 4 classes), with mean entropy of the color-based model set to a value 3 times larger than the mean entropy of the texture-based model. (k) Variation $\Delta D$ of model descriptiveness as a function of the number of classes. 


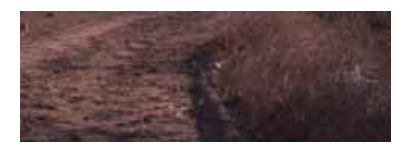

(a)

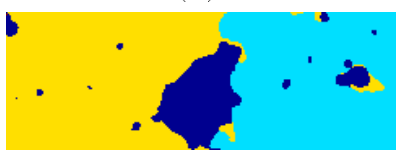

(c)

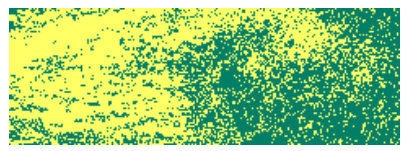

(b)

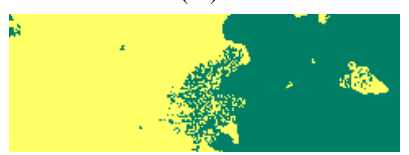

(d)
Figure 3: (a): Test image. (b) Color-based supervised classification into the "road" class (yellow) and the "grass" area (green.) (c) Texture-based unsupervised segmentation (3 classes.) (d) Hybrid supervised/unsupervised classification.

same contribution to the final segmentation. This hypothesis does not hold true if the "softness" of segmentation is very different in the two models. The softness of segmentation can be measured in terms of the mean entropy of the model, a well-known concept in the fields of statistical physics and mixture estimation [4],[6].

Given a feature $z$, the entropy of the posterior distribution $P(j \mid z)$ is defined by [2]

$$
s(z)=-\sum_{j=1}^{N} P(j \mid z) \log P(j \mid z)
$$

The entropy $s(z)$ measures the softness of the class assignment. A distribution with null entropy assigns $z$ to exactly one class; the maximum value of the entropy is $\log N$, and is attained when all classes are equally likely to have generated $z$. The mean entropy $S$ of a model is defined by the expectation of $s(z)$ computed with respect to $p(z)$ :

$$
S=-\sum_{j=1}^{N} E[P(j \mid z) \log P(j \mid z)]
$$

In practice, the mean entropy can be estimated by averaging $s(z)$ over the observed image. A model with null mean entropy can only perform "hard" classification, and will be called degenerate. Note that the mean entropy of a model estimated via Expectation Maximization is a function of the "temperature" of the algorithm [6].

It is easy to see that if two models to be merged have very different values of the mean entropy, the model with the smallest entropy will "dominate" the combined model. This undesirable effect may be corrected by applying to one of the two models the simple entropy equalization procedure proposed in the following.

Our equalization operator starts from a model $\mathcal{M}$ and produces a new model with the same number of classes $N$. The entropy of this new model can be tuned to match any desired value $S_{0}<\log N$, and the associated Bayesian classifier yields the same results as the Bayesian classifier for $\mathcal{M}$. The equalization operator simply replaces each posterior distribution $P(j \mid z)$ with the new distribution $P^{e q}(j \mid z)$ defined as follows:

$$
P^{e q}(j \mid z)=c(z) P(j \mid z)^{\alpha}, \alpha \geq 0
$$

where $c$ is a normalizing coefficient:

$$
c(z)=\frac{1}{\sum_{j=1}^{N} P(j \mid z)^{\alpha}}
$$

The mean entropy properties of the equalization operator are summarized by the following result:

Fact 2 Equalization decreases the mean entropy of a non-degenerate model if $\alpha>1$, and increases it if $\alpha<1$.

The proof can be found in the Appendix. Note that $\alpha=0$ implies that the mean entropy of $P^{e q}(j \mid z)$ is equal to $\log N$; the mean entropy of $P^{e q}(j \mid z)$ can be made as small as desired by a suitably large value of $\alpha$. Also note that for each feature $z$ the location of the maximum of the posterior distribution is not changed by the equalization, so that the Bayesian classifier will yield the same segmentation for the two models.

Now, suppose that the two models to be merged have different mean entropies. We may modify one of the models via the equalization operator, so that its mean entropy matches the mean entropy of the other model. The appropriate value of the parameter $\alpha$ may be found using any non-linear one-dimensional minimization technique.

In some cases, equalization may also be used to make either of the two models dominant, i.e. to assign different "weights" to the models to be merged. For example, figure $2(\mathrm{j})$ shows the results of Bayesian segmentation after equalizing the color-based model to a value of the mean entropy 3 times larger than the mean entropy of the texture-based model (the combined model dimension was reduced to 4 by classclipping.) By comparing figure $2(\mathrm{j})$ with (h) and (c) it results clear that the final segmentation is dominated by the texture-based model. We should point out, however, that while this and other experimental results are very encouraging, we still lack a complete understanding of the relation between mean entropy and model dominance, which will be the object of future research. 


\section{Hybrid classification}

The main differences between supervised classification and unsupervised clustering can be summarized as follows:

1. The classes ("labels") of a supervised classifier usually represent "physical" causes, and therefore are not logically interchangeable;

2. The statistical model of a supervised classifier is usually learned from training data, while unsupervised clustering does not require training in principle.

The Bayesian classifier assigns a feature $z$ to the maximizer of the posterior distribution [2]. In many instances, only the conditional likelihoods $p(x \mid j)$ are learned; however, reasonable assumptions about the class priors $P(j)$ are often available, and the posterior distributions can be computed using Bayes' rule.

In this section we propose to merge a model $\mathcal{M}^{s}$ for supervised classification with a model $\mathcal{M}^{u}$ for unsupervised segmentation (based on a different feature space,) to create a "hybrid" classifier which assigns each image point to some label of $\mathcal{M}^{s}$. The intuition is that information from the "unsupervised model" (which identifies clusters in the feature space based on the current image) may be used to leverage the classification performed by the "supervised model", which is learned from a large training data set and may not be optimal for the current instance.

The merging algorithm discussed in the previous sections defines a model $\mathcal{M}$ with classes that are the union of elements of the Cartesian product of $\mathcal{M}^{s}$ and $\mathcal{M}^{u}$. If $\mathcal{C}$ represents a generic class of $\mathcal{M}$, we may write

$$
\mathcal{C}=\bigcup_{v \in V} \bigcup_{w(v)}\left(\mathcal{C}_{v}^{s}, \mathcal{C}_{w(v)}^{u}\right)
$$

where $\mathcal{C}^{s}$ and $\mathcal{C}^{u}$ are classes of $\mathcal{M}^{s}$ and $\mathcal{M}^{u}$ respectively, indexed by the corresponding subscripts. To complete the definition of the hybrid classification model, we need to be able to assign labels from $\mathcal{M}^{s}$ to the image using the new super-model. In other words, we need to identify each class $\mathcal{C}$ with some class of $\mathcal{C}^{s}$. If the set $V$ of classes of $\mathcal{M}^{s}$ that form the super-class $\mathcal{C}$ is composed by just one element $v$, than we simply identify $\mathcal{C}$ with $\mathcal{C}_{v}^{s}$. In general, however, $V$ may have more than one element; in this case, we identify $\mathcal{C}$ with the class $\mathcal{C}_{v}^{s}$ that maximizes the contribution to $\mathcal{C}$, defined by

$$
E\left[P\left(\bigcup_{w(v)}\left(\mathcal{C}_{v}^{s}, \mathcal{C}_{w(v)}^{u}\right) \mid z\right)\right]=
$$

$$
=E\left[P_{s}\left(v \mid z_{s}\right) \sum_{w(v)} P_{u}\left(w(v) \mid z_{u}\right)\right]
$$

where the expectation is computed with respect to $p(z)$ (with $\left.z=\left(z_{s}, z_{u}\right).\right) \quad P_{s}(\cdot \mid \cdot), P_{u}(\cdot \mid \cdot)$ and $P(\cdot \mid \cdot)$ represent the posteriors of the models $\mathcal{M}_{s}, \mathcal{M}_{u}$ and $\mathcal{M}$ respectively.

We present an example of hybrid classification in Figure 3. Figure 3(a) shows a scene with a dirt road on the left and dry grass on the right. Supervised colorbased classification (figure 3(b)) is performed using a trained gaussian model. The "road" class and the "grass" class have very similar colors; this is the reason why pixels in the top-right quadrant are misclassified as belonging to the "road" class. Figure 3(c) shows the results of unsupervised texture segmentation with three classes, computed via Expectation Maximization. The segmenter isolates uniform regions corresponding to the road and to the grass areas, plus a region corresponding to the border of the road. After mean entropy equalization, the two models are merged into a new model with four classes; the final hybrid classification is shown in Figure $3(\mathrm{~d})$. The hybrid classifier has correctly labeled each one of the four classes of the merged model as either "road" or "grass". The information from the texture model has helped to correctly classify most pixels that were misclassified in figure $3(\mathrm{~b})$.

\section{Conclusions}

We have presented a technique for merging together two segmentations computed independently over color and texture. Our technique is very general, and in principle can be applied also to other classes of features, such as motion; it only requires that the posterior distributions that originated the segmentations are available. The results show the effectiveness of the maximum descriptiveness criterion for reducing the dimensionality of the Cartesian product of the two mixture models. We have also introduced a technique for hybrid supervised/unsupervised classification, based on our merging algorithm, that can improve the performance of supervised classification using consensus from different features.

\section{Acknowledgments}

The research described in this paper was carried out by the Jet Propulsion Laboratory, California Institute of Technology, under a contract with the National Aeronautics and Space Administration. This work was supported in part by the NASA Telerobotics program and by the NASA Remote Exploration and Experimentation task. Reference herein to any specific commercial product, process, or service by trade 
name, trademark, manufacturer, or otherwise, does not constitute or imply its endorsement by the United States Government or the Jet Propulsion Laboratory, California Institute of Technology.

This work was originated by discussions with Eric Mjolsness and Becky Castano. Matt Klimesh is kindly acknowledged for fruitful feedback.

\section{Appendix}

Proof of Fact 1. A class-clipping operation can always be implemented by a sequence of class-clippings involving two classes at a time. We show in the following that the model descriptiveness can never increase with any such step. Assume classes $j$ and $k$ are clipped together; using (7) and (6), and remembering that the likelihood $p(z)$ does not change after class-clipping, we maintain that the variation $\Delta D$ of the model descriptiveness is

$$
\begin{aligned}
& \Delta D= \frac{\int(P(j \mid z)+P(k \mid z))^{2} p(z) d z}{P(j)+P(k)}- \\
&-\frac{\int P(j \mid z)^{2} p(z) d z}{P(j)}-\frac{\int P(k \mid z)^{2} p(z) d z}{P(k)} \\
&=\int\left(\frac{(P(j \mid z)+P(k \mid z))^{2}}{P(j)+P(k)}-\frac{P(j \mid z)^{2}}{P(j)}-\frac{P(k \mid z)^{2}}{P(k)}\right) p(z) d z
\end{aligned}
$$

Now, it is easy to prove that, for any $z$, term $\frac{(P(j \mid z)+P(k \mid z))^{2}}{P(j)+P(k)}-\frac{P(j \mid z)^{2}}{P(j)}-\frac{P(k \mid z)^{2}}{P(k)}$ is always nonpositive. Thus, since $p(z)$ is always non-negative, $\Delta D \leq 0$, and the claim is proved.

Proof of Fact 2. We just need to prove the claim for the case $\alpha<1$. The proof is based on the following two results.

Lemma 1. The entropy of a probability distribution increases if two values of the distribution are moved closer to each other, while the other values are left untouched.

Proof. The claim is a direct consequence of the convexity of the function $-x \log x$.

Lemma 2. Let $P(j), 1 \leq j \leq N$ be a probability distribution and, for a given $K<N$, let $J_{1}$ and $J_{2}$ be the sets of the indices of the $K$ smallest values and of the $N-K$ largest values of $P(j)$ respectively. Now form a new distribution $\bar{P}(j)$ from $P(j)$ by increasing some of the values with index in $J_{1}$ while at the same time decreasing some of the values with index in $J_{2}$, with the requirement that

$$
\max \left\{\bar{P}(j), j \in J_{1}\right\} \leq \min \left\{\bar{P}(i), i \in J_{2}\right\}
$$

Then the entropy of $\bar{P}(j)$ is higher than the entropy of $P(j)$.
Proof. The transformation from $P(j)$ to $\bar{P}(j)$ can be decomposed into a sequence of steps, each one involving just one value with index in $J_{1}$ and just one value with index in $J_{2}$. Therefore, by Lemma 1, the entropy is increased at each such step.

Now, it is easy to prove that the function $c(z) x^{\alpha}-x$, with $c(z)$ defined in (11), vanishes in correspondence of the value $x=c(z)^{\alpha-1}$, which is located between the smallest and the largest values of $P(j \mid z)$. Therefore, if $P(j \mid z)$ has non-null entropy, the equalization operator (10) with $\alpha<1$ falls into the class of transformations considered in Lemma 2: the set $J_{1}$ is composed by all the $j$ such that $P(j \mid z) \leq c(z)^{\alpha-1}$, the set $J_{2}$ is composed by all the other indices. This proves that for any $z$ the entropy of $P(j \mid z)$ increases as a consequence of equalization with $\alpha<1$.

\section{References}

[1] S. Belongie, C. Carson. H. Greenspan, J. Malik. Color- and texture-based image segmentation using EM and its application to content-based image retrieval. 675-682, 6th ICCV, New Delhi, India, January 1998.

[2] C.M. Bishop. Neural networks for pattern recognition. Clarendon Press, Oxford, U.K., 1995.

[3] B.S. Manjunath and W.Y. Ma. Texture features for browsing and retrieval of image data. IEEE Trans. Pattern Anal. Mach. Intell., 18(8):837-842, August 1996.

[4] R.M. Neal and G.E. Hinton. A new view of the EM algorithm that justifies incremental and other variants. Submitted to Biometrika.

[5] Y. Rubner, L. Guibas, and C. Tomasi. The earth mover's distance, multi-dimensional scaling, and color-based image retrieval. Proc. ARPA Image Understanding Workshop, May 1997.

[6] Y. Weiss and E.H. Adelson. A unified mixture framework for motion segmentation: incorporating spatial coherence and estimating the number of models. Proc. IEEE CVPR'96, 321-326, San Francisco, 1996. 